\title{
铝合金大气腐蚀现场暴露试样的图像分析
}

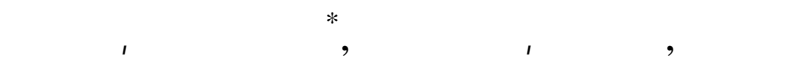

(1) 天津大学材料科学与工程学院, 天津 300072;

(2) 北京航空材料研究院, 北京 100095;

(3) 中国科学院金属研究所, 金属腐蚀与防护国家重点实验室, 沈阳 110016

* 联系人, E-mail: szsong@tju.edu.cn

2008-01-03 收稿, 2008-04-09 接受

国家自然科学基金重大项目(批准号: 50499335)资助

摘要 构建了适用于现场暴露试样的腐蚀形貌图像采集系统, 并应用该系统在北京大气腐蚀试验 站对铝合金腐蚀初期的形貌图像进行跟踪采集. 所得图像经过降噪处理后, 应用小波变换进行分 解并提取子图像的能量值. 根据图像能量值的变化, 对试样的腐蚀程度进行了定性和定量判断, 实验结果与腐蚀失重数据分析所得结论基本一致.

关键词 大气腐蚀 铝合金 腐蚀形貌 图像分析
材料的大气腐蚀是一个普遍存在的现象. 传统 的重量法虽然一直是评价金属大气腐蚀速率的重要 方法, 但对局部腐蚀以及早期腐蚀行为存在一定局 限性. 表征金属材料腐蚀特性除腐蚀速率等数据外, 表面腐蚀形貌也是评价耐蚀性能的重要特征之一. 图像分析技术是表征腐蚀破坏初始阶段的有效工具. 近年来, 数字图像处理技术和数学理论的结合, 特别 是小波图像分析技术的发展，为材料表面腐蚀形貌 图像分析注入了新的活力, 这也给金属材料大气腐 蚀研究带来新的思路和手段 $[1 \sim 5]$. 宋诗哲等人以大气 暴露纯锌试样 ${ }^{[6,7]}$ 以及模拟加速腐蚀的铝合金试样 ${ }^{[8,9]}$ 为研究对象, 分别建立了适用于实验室的腐蚀形貌 图像采集系统并提取材料腐蚀形貌特征, 对图像分 析在腐蚀科学中的应用进行了初步的研究.

本文以已有工作为基础，构建适用于现场暴露 试样的腐蚀形貌图像采集系统, 运用该系统对大气 暴露现场铝合金试样腐蚀初期的形貌进行跟踪采集, 通过图像处理分析, 研究了铝合金大气腐蚀的早期 行为特征, 并对腐蚀程度进行了定性和定量判断. 本 文工作将数字图像分析技术在腐蚀科学中的应用推 向深入, 对于金属材料大气腐蚀现场监测具有重要 意义。

\section{1 铝合金大气腐蚀试样图像采集的实现}

(i) 实验试样. 测试现场为北京大气腐蚀试验 站. 实验材料为 2004 年 11 月投放的 5 种新型铝合金, 表 1 列出了铝合金试样编号及暴露 1 周期(1 年)后测 得的腐蚀失重数据. 不同型号铝合金各有 20 片平行 试样, 每个暴露周期有 4 片平行试样: 3 片用于腐蚀 失重测量, 1 片用于腐蚀产物分析. 5 种铝合金试样各 选取其中 3 片作为图像跟踪采集的平行样本. 利用腐 蚀形貌图像采集系统, 分别对暴露 207,325, 539 和 710 天的铝合金试样腐蚀形貌进行了跟踪采集. 所得 图像编号记为 “*-铝合金代号-平行试样编号”, 其中 “*”为 $0,1,2,3,4$, 分别代表未腐蚀试样、暴露 207, 325, 539 和 710 天的腐蚀形貌图像. $\mathrm{a}$ 和 $\mathrm{b}$ 分别表示未 腐蚀铝合金试样的正反两面.

表 1 铝合金试样编号及暴露 1 年腐蚀失重数据

\begin{tabular}{cccc}
\hline 铝合金代号 & 平行试样编号 & 热处理状态 & 腐蚀失重 $/ \mathrm{g} \cdot \mathrm{m}^{-2}$ \\
\hline B06 & & $\mathrm{T} 4$ & 0.9588 \\
B19 & & $\mathrm{AT}$ & 0.9437 \\
$\mathrm{~B} 7 \mathrm{~B} 6$ & $1 \sim 20$ & $\mathrm{~T} 6$ & 0.9048 \\
$\mathrm{~B} 7 \mathrm{~B} 7$ & & $\mathrm{~T} 74$ & 0.6858 \\
$\mathrm{~B} 952$ & & $\mathrm{~T} 2$ & 0.8586 \\
\hline
\end{tabular}



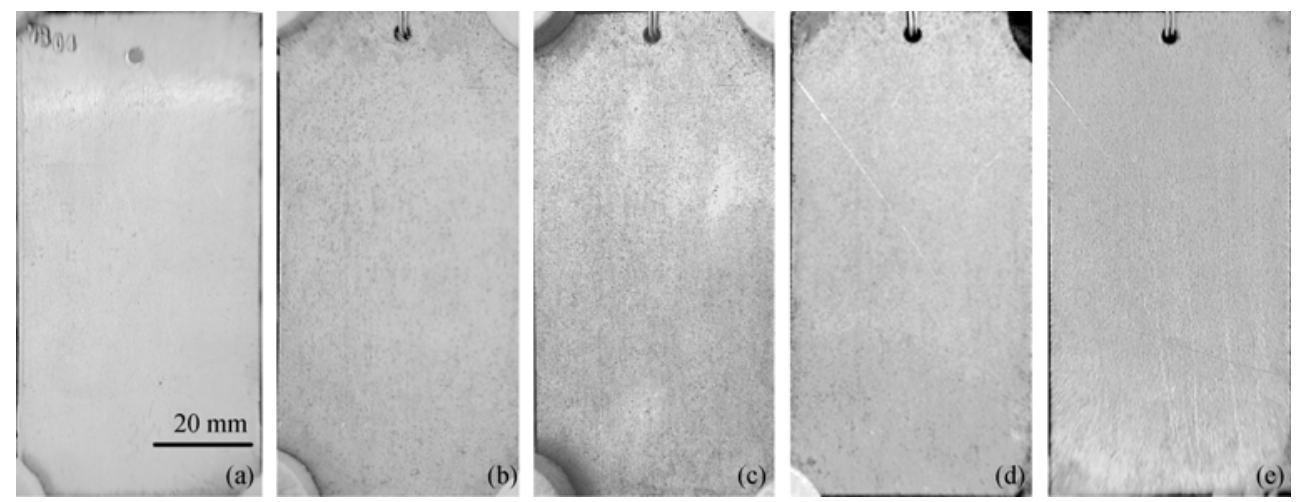

图 1 采集的部分铝合金腐蚀形貌图像

(a) 0-B7B6-a; (b) 1-B7B6-12; (c) 2-B7B6-12; (d) 3-B7B6-12; (e) 4-B7B6-12

(ii) 现场图像采集系统的建立及腐蚀形貌图像 的采集. 现场图像采集系统由 HIROX 公司生产的 KH-3000 三维视频显微镜主机、0-40×低倍率变焦镜 头、CRT 显示器以及三脚架构成. 主要性能指标为: 内置 211 万像素的 CCD, 输出数字图像为模拟 RGB UXGA (1600×1200 dpi). 图 1 为采集的部分铝合金腐 蚀形貌图像.

\section{2 铝合金试样大气腐蚀形貌的图像分析方法}

\section{1 腐蚀形貌图像的预处理}

为消除腐蚀产物的影响, 同时考虑到化学处理 方法会影响试样的腐蚀形貌图像, 因此图像采集在 化学法去除腐蚀产物前进行, 利用软毛刷去除试样 表面浮尘及杂物, 而腐蚀失重仍在去除腐蚀产物后 测得. 用中值滤波法对所得图像进行预处理, 滤除图 像中的噪声信号. 考虑到试样边角及固定孔的影响, 图像处理区域应进行适当选取, 以图像垂直对称轴 为所选区域的对称轴, 截取 $50 \mathrm{~mm} \times 25 \mathrm{~mm}$ 的矩形区 域作为图像处理区. 腐蚀形貌图像预处理及处理区 域选取方法参见文献[8].

\section{2 基于小波分解的图像分析}

选用DB小波系的Haar小波，对选取的腐蚀形貌 图像处理区域进行 3 水平小波分解 ${ }^{[7]}$. 以小波分解每 个子图像的能量值 $E$ 作为腐蚀形貌特征, 计算式为

$$
E=\sum_{i=0}^{255} H(i)^{2},
$$

式中 $H(i)$ 为图像中像素值为 $i$ 的点占总像素数的比值. $E_{\mathrm{H} 1}, E_{\mathrm{H} 2}, E_{\mathrm{H} 3}$ 和 $E_{\mathrm{V} 1}, E_{\mathrm{V} 2}, E_{\mathrm{V} 3}$ 以及 $E_{\mathrm{D} 1}, E_{\mathrm{D} 2}, E_{\mathrm{D} 3}$ 分别为 水平方向、垂直方向和对角方向进行 1, 2, 3 水平小波 变换后得到的子图像的能量值.

\section{3 铝合金试样大气腐蚀形貌的图像分析}

试样腐蚀形貌图像分析，以 B06 铝合金为例. 表 2 列出了 B06 铝合金腐蚀形貌图像小波分解子图像的 能量值. 图 2 为不同暴露时期 B06 铝合金子图像能量 值分布, 其数值分别为平行试样的子图像能量值的 均值.

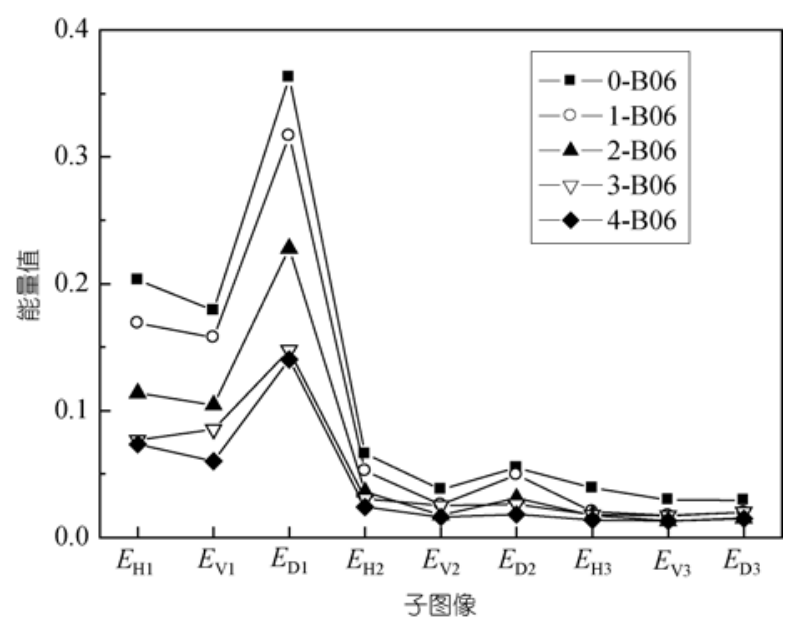

图 2 B06 铝合金试样不同腐蚀阶段能量值分布

由图 2 可以对 B06 铝合金试样的腐蚀程度进行 定性的判断, 大气腐蚀相对严重的试样(如 3-B06 和 4-B06), 其相应的子图像的能量值都较低; 而腐蚀相 对轻微或未腐蚀试样(如 0-B06 和 1-B06), 对应的子 图像的能量值都较高. 用同样的方法, 可以得到 B19, B7B6，B7B7 和 B952 铝合金试样的不同腐蚀时期能 量值的分布, 见图 3. 由图 3 可以看出, 各牌号铝合金 试样腐蚀形貌图像的子图像能量值, 均呈现暴露时 间增加、能量值降低的趋势, 即腐蚀越严重, 能量值 越低. 因此，可通过腐蚀形貌图像的子图像能量值对 
表 2 B06 铝合金腐蚀形貌图像小波分解子图像的能量值

\begin{tabular}{|c|c|c|c|c|c|c|c|c|c|}
\hline \multirow{2}{*}{ 试样 } & \multicolumn{9}{|c|}{ 特征值 } \\
\hline & $E_{\mathrm{H} 1}$ & $E_{\mathrm{V} 1}$ & $E_{\mathrm{D} 1}$ & $E_{\mathrm{H} 2}$ & $E_{\mathrm{V} 2}$ & $E_{\mathrm{D} 2}$ & $E_{\mathrm{H} 3}$ & $E_{\mathrm{V} 3}$ & $E_{\mathrm{D} 3}$ \\
\hline 0-B06-a & 0.2238 & 0.1826 & 0.3778 & 0.0708 & 0.0311 & 0.0606 & 0.0417 & 0.0242 & 0.0259 \\
\hline 0-B06-b & 0.1824 & 0.1755 & 0.3476 & 0.0611 & 0.0447 & 0.0495 & 0.0363 & 0.0352 & 0.0329 \\
\hline 1-B06-13 & 0.1805 & 0.1622 & 0.3578 & 0.0539 & 0.0256 & 0.0477 & 0.0195 & 0.0149 & 0.0178 \\
\hline 1-B06-14 & 0.1577 & 0.1379 & 0.2320 & 0.0471 & 0.0244 & 0.0423 & 0.0189 & 0.0209 & 0.0222 \\
\hline 1-B06-15 & 0.1684 & 0.1728 & 0.3601 & 0.0567 & 0.0273 & 0.0580 & 0.0229 & 0.0161 & 0.0192 \\
\hline 2-B06-13 & 0.1158 & 0.0984 & 0.2400 & 0.0355 & 0.0180 & 0.0296 & 0.0120 & 0.0123 & 0.0125 \\
\hline 2-B06-14 & 0.1015 & 0.1146 & 0.2512 & 0.0347 & 0.0161 & 0.0312 & 0.0148 & 0.0124 & 0.0146 \\
\hline 2-B06-15 & 0.1243 & 0.1004 & 0.1929 & 0.0376 & 0.0192 & 0.0322 & 0.0260 & 0.0142 & 0.0188 \\
\hline 3-B06-13 & 0.0760 & 0.0827 & 0.1496 & 0.0274 & 0.0224 & 0.0271 & 0.0166 & 0.0175 & 0.0166 \\
\hline 3-B06-14 & 0.0799 & 0.1015 & 0.1598 & 0.0354 & 0.0340 & 0.0294 & 0.0243 & 0.0220 & 0.0244 \\
\hline 3-B06-15 & 0.0744 & 0.0716 & 0.1334 & 0.0286 & 0.0193 & 0.0224 & 0.0131 & 0.0121 & 0.0195 \\
\hline 4-B06-13 & 0.0740 & 0.0578 & 0.1419 & 0.0239 & 0.0161 & 0.0171 & 0.0149 & 0.0130 & 0.0156 \\
\hline 4-B06-14 & 0.0703 & 0.0575 & 0.1326 & 0.0237 & 0.0167 & 0.0176 & 0.0135 & 0.0140 & 0.0115 \\
\hline 4-B06-15 & 0.0760 & 0.0657 & 0.1465 & 0.0245 & 0.0157 & 0.0195 & 0.0136 & 0.0122 & 0.0174 \\
\hline
\end{tabular}
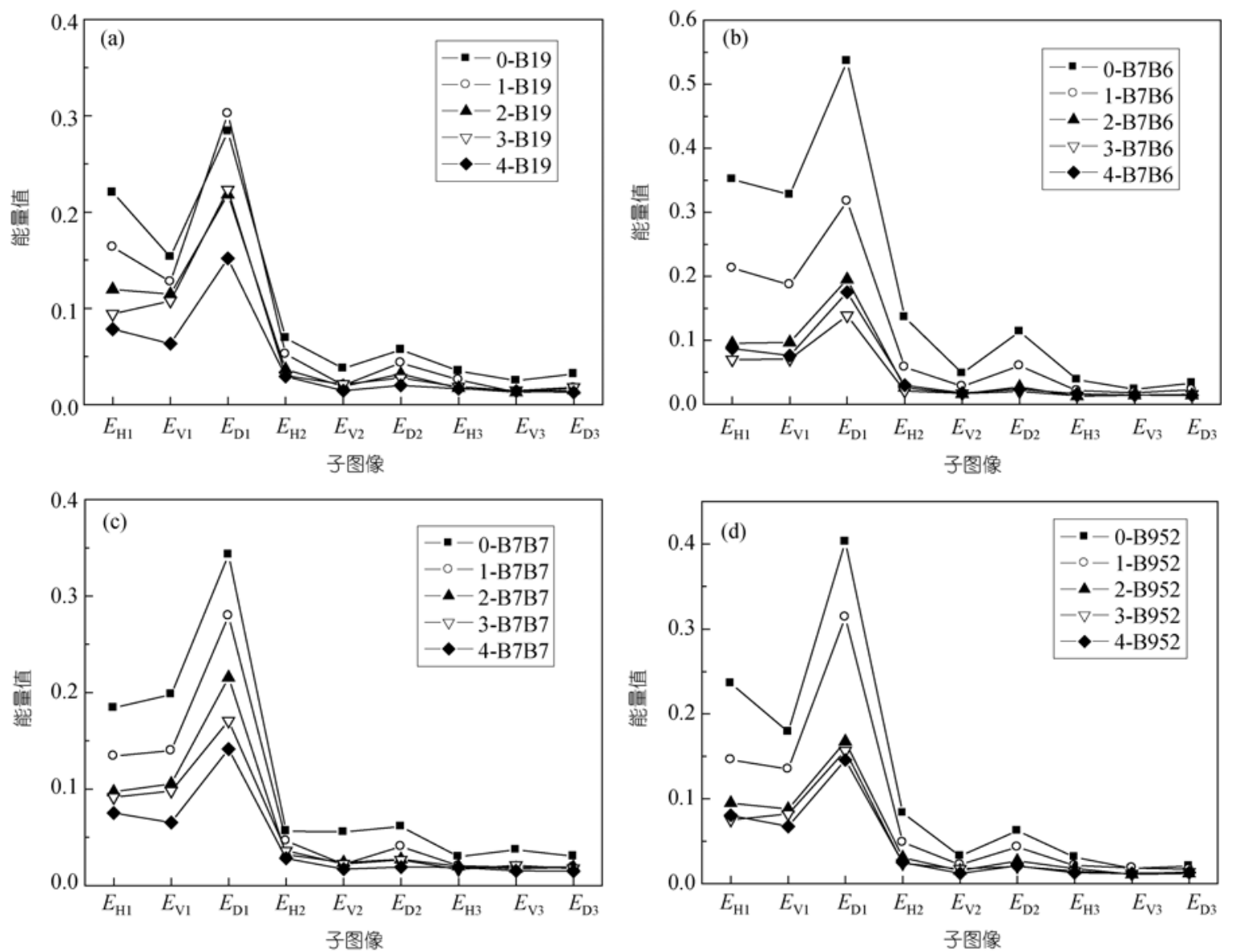

图 3 编号 B19(a), B7B6(b), B7B7(c), B952(d)铝合金试样不同腐蚀阶段能量值分布

同种材料铝合金试样腐蚀程度进行定性的判断.

以铝合金腐蚀形貌图像能量值的损失量 $D$ 作为 评价铝合金试样腐蚀损失的参数. 计算公式如下:

$$
E_{i}=E_{\mathrm{H} 1}+E_{\mathrm{V} 1}+E_{\mathrm{D} 1}+E_{\mathrm{H} 2}+E_{\mathrm{V} 2}+E_{\mathrm{D} 2}
$$

$$
\begin{gathered}
+E_{\mathrm{H} 3}+E_{\mathrm{V} 3}+E_{\mathrm{D} 3}, i=0,1,2,3,4, \\
D_{i}=E_{0}-E_{i}, i=1,2,3,4,
\end{gathered}
$$

其中 $i$ 表示不同腐蚀阶段, $E_{i}$ 为不同腐蚀阶段采集图 像的小波分解子图像的能量值总和. 
图 4 是五种铝合金暴露不同时间的能量值损失, 按幂函数 $D=A t^{n}$ ( $A, n$ 为常数)进行回归分析得出的 图像能量值损失 $D$ 与暴露时间 $t$ 的幂函数曲线, 回归 系数见表 3. 图 4 中的回归曲线和表 3 中的回归系数 表明, 铝合金试样的回归方程有较好的相关性. 回归 分析得到的 $n$ 值受材料的特性和环境因素影响, $n$ 值 越大反映铝合金腐蚀图像能量损失的变化率越大, 即铝合金腐蚀损失的速率越大. 5 种铝合金的回归参 数 $n$ 值都小于 1 , 铝合金腐蚀形貌图像的能量值损失 量变化, 随暴露腐蚀时间延长逐渐下降, 说明暴露

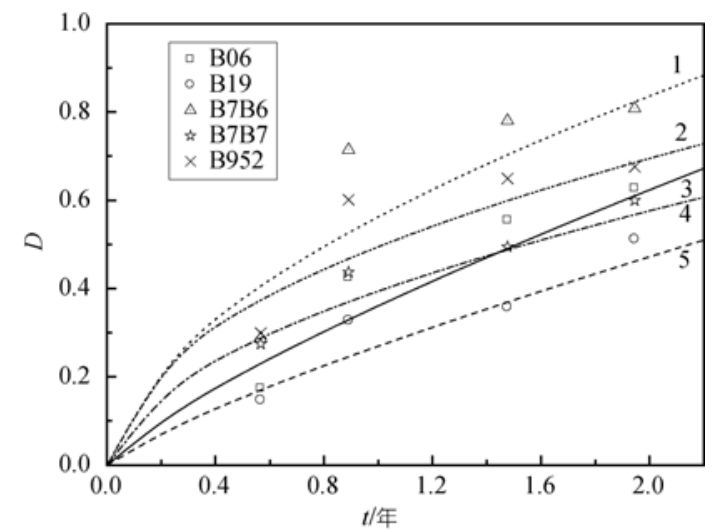

图 4 铝合金腐蚀形貌图像能量值损失 $D$ 与暴露时间 $t$ 的 回归曲线

1, B7B6; 2, B952; 3, B06; 4, B7B7; 5, B19

表 3 图像能量值损失 $D$ 与暴露时间 $\boldsymbol{t}$ 的回归参数

\begin{tabular}{cccc}
\hline 铝合金代号 & $A$ & $n$ & 相关系数 $R$ \\
\hline B06 & 0.3893 & 0.7878 & 0.9482 \\
B19 & 0.2911 & 0.8071 & 0.9454 \\
B7B6 & 0.5951 & 0.5695 & 0.8455 \\
B7B7 & 0.4321 & 0.3665 & 0.9179 \\
B952 & 0.5193 & 0.4869 & 0.8617 \\
\hline
\end{tabular}

初期铝合金腐蚀产物对试样起到较好的保护作用, 抑制了腐蚀加速发展.

根据前面的回归分析结果, 推得图像能量值损 失 $D$ 的变化速率为 $\mathrm{d} D / \mathrm{d} t=A n \cdot t \cdot n^{-1}$, 即腐蚀损失的 速率取决于 $A \cdot n$ 和 $t^{n-1}$ 两项, 其中 $t^{n-1}$ 项的大小与试 样暴露时间有关, 而常数项 $A \cdot n$ 则可作为考察五种 铝合金大气腐蚀速率的指标. 铝合金试样的 $A \cdot n$ 值 见表 4, 北京大气试验站现场暴露的五种铝合金中编 号 B06 和 B7B6 的 $A \cdot n$ 值相对较大, 腐蚀速率较大, 而编号 B7B7 的 $A \cdot n$ 值相对较小, 腐蚀速率最小, 最 耐蚀. 所得结论与北京大气腐蚀试验站提供的腐蚀 失重数据(见表 1)基本一致.

表 4 铝合金试样的 $A \cdot n$ 值

\begin{tabular}{cccccc}
\hline 铝合金代号 & B06 & B19 & B7B6 & B7B7 & B952 \\
\hline$A \cdot n$ 值 & 0.3067 & 0.2350 & 0.3389 & 0.1584 & 0.2529 \\
\hline
\end{tabular}

\section{4 结论}

(i) 构建的现场图像采集系统适用于大气腐蚀 现场暴露试样的图像采集, 并应用该系统对铝合金 试样大气腐蚀初期的形貌图像进行了跟踪采集.

(ii ) 运用小波分解的方法, 对铝合金试样大气 腐蚀初期的腐蚀形貌图像进行分析并提取子图像的 能量值. 根据能量值的变化对试样的腐蚀程度进行 了定性和定量判断. 研究表明, 同种型号铝合金试样 在不同腐蚀阶段均呈现暴露时间增加、能量值降低的 趋势. 五种铝合金试样中, 编号 B06 和 B7B6 铝合金 相对腐蚀速率较大, 而编号 B7B7 铝合金腐蚀速率最 小, 最耐蚀. 其结果与根据腐蚀失重数据分析所得结 论基本一致.

\section{参考文献}

1 Silva J W J, Bustamante A G, Codaro E N, et al. Morphological analysis of pits formed on Al 2024-T3 in chloride aqueous solution. Appl Surf Sci, 2004, 236(1-4): 356-365[ [doi]

2 Codaro E N, Nakazato R Z, Horovistiz A L, et al. An image processing method for morphology characterization and pitting corrosion evaluation. Mater Sci Eng A, 2002, A334(1-2): 298-306

3 Frantziskonis G N, Simon L B, Woo J, et al. Multiscale characterization of pitting corrosion and application to an aluminum alloy. Eur J Mech A: Solids, 2000, 19(2): 309-318[ [doi]

4 Itzhak D, Dinstein I, Zilberberg T. Pitting corrosion evaluation by computer image processing. Corr Sci, 1981, 21: 17-22[doi]

5 Journaux S, Guillaumin C, Gouton P, et al. Image analysis of corrosion pit damage. Opt Eng, 1999, 38(8): 1312-1318 [doi] 宋诗哲，王守琰，高志明，等。图像识别技术研究有色金属大气腐蚀早期行为。金属学报, 2002, 38: 893-896

Wang S Y, Song S Z. Image analysis of atmospheric corrosion exposure of zinc. Mater Sci Eng A, 2004, 385: 377-381

宋诗哲，陶蕾，王守琰，等. LC4CS 铝合金模拟加速腐蚀试样的图像分析. 金属学报, 2006, 42: 426一-430

Tao L, Song S Z, Wang S Y, et al. Image analysis of periodic rain accelerated corrosion of aeronautical aluminium alloys. Mater Sci Eng A, 2008, 476: 210-216 $\underline{\text { [doi] }}$ 